\title{
PAPERS
}

\section{Nocturnal oesophageal motor activity is dependent on sleep stage}

\author{
F Castiglione, C Emde, D Armstrong, C Schneider, P Bauerfeind, G Stacher, A L Blum
}

\begin{abstract}
Simultaneous overnight oesophageal pH and manometric and sleep electroencephalographic recordings were performed in eight healthy subjects, aged 20-38 years, to test the hypothesis that the frequency of primary, swallow related contractions decreases progressively with deeper sleep stages whereas the frequency of secondary contractions remains constant throughout the night. During the nocturnal period ( 2300 to 0700 ), periods of oesophageal motor quiescence were interspersed by clusters of contractions detected 5 and $15 \mathrm{~cm}$ above the lower oesophageal sphincter. Primary contractions decreased in frequency from $1.42 / \mathrm{min}$ (median) during arousal periods to $0.22 / \mathrm{min}$ during stage 1 sleep, $0.05 / \mathrm{min}$ during stages 2 to 4 combined, and $0.03 / \mathrm{min}$ during rapid eye movement (REM) sleep. Secondary contractions were also most frequent during arousal periods $(0.51 / \mathrm{min})$ and they, too, decreased in frequency during stage $1(0.35 / \mathrm{min})$ and stages 2 to 4 combined (0.08/min). During REM sleep, however, the frequency of secondary contractions increased $(0.50 / \mathrm{min})$ to levels noted during arousal and stage 1 sleep. Compared with primary contractions, secondary contractions had a lower amplitude $(51.9 \mathrm{hPa} v \mathbf{7 6 . 0}$ $\mathrm{hPa} ; \mathrm{p}=0.0078)$ and a shorter duration (3.08 $v$ $4.06 \mathrm{~s} ; \mathrm{p}=0.0078$ ). The results of this study suggest that there is no intrinsic oesophageal motor activity in the absence of a stimulatory input from the central nervous system and that the increased number of secondary contractions during REM sleep may be a result of an REM related increase in autonomic nervous system activity although a temporary decrease of efferent inhibitory influences cannot be ruled out. Nocturnal contraction clusters comprise both primary contractions during arousals and stage 1 sleep and secondary contractions during REM sleep.

(Gut 1993; 34: 1653-1659)
\end{abstract}

Oesophageal motor activity may be modulated both by central factors, such as psychological stress $^{1}$ and peripheral factors, such as the size, viscosity, and temperature of a swallowed bolus, but it is difficult to differentiate clearly between the participation of the central and peripheral modulators. If, however, one assumes that central processes, and particularly those under voluntary control, become less important as a subject progresses from wakefulness to deep sleep, one could attribute any residual oesophageal activity during deep sleep to peripheral mechanisms. This study was designed to investigate the dependence of normal, nocturnal oesophageal motor activity on the state of central nervous system arousal. Specifically, the hypotheses tested were firstly, that primary, swallow related contractions decrease in frequency as the depth of sleep progresses from stage 1 (light sleep) to stage 4 (deep sleep) and secondly, that secondary contractions, unrelated to swallowing, are comparably frequent during all sleep stages because they are initiated by local stimuli, independently of any central events.

The hypotheses were tested by making simultaneous overnight oesophageal $\mathrm{pH}$, manometric and electroencephalographic recordings to assess whether oesophageal motility varies with respect to sleep stages or the occurrence of gastrooesophageal reflux.

\section{Methods and subjects}

\section{SUBJECTS AND STUDY PROTOCOL}

Eight healthy subjects, three women and five men (median age 24 years, range $20-38$ ), all of whom received remuneration for their participation, were studied. No subject had a history of gastrointestinal or other intercurrent illness, oesophageal symptoms or sleep disturbances and none was receiving any treatment. All subjects gave written informed consent; the study was conducted with the approval of the local ethical committee in accordance with the Declaration of Helsinki.

One week before the recordings, a routine manometric examination was performed in each subject, using a low compliance perfused capillary system ${ }^{3}$ to localise the lower oesophageal sphincter and to exclude oesophageal motor abnormalities. For the nocturnal recordings, the subjects arrived in the laboratory at 1700 having fasted from 1200 . The combined oesophageal $\mathrm{pH}$ and manometric recording assembly was passed transnasally and advanced until it reached the stomach. A rapid pull through was performed to confirm the position of the lower oesophageal sphincter and the assembly was positioned such that the $\mathrm{pH}$ electrode and the more distal of the two pressure transducers were located $5 \mathrm{~cm}$ above the lower oesophageal 
sphincter, while the more proximal pressure transducer was $15 \mathrm{~cm}$ above the lower oesophageal sphincter. The $\mathrm{pH}$ and manometric recordings started at 1800 and lasted for 18 hours; the subjects ate standardised meals at 1900 and 0730. The electroencephalogram, electrooculogram, pharyngeal electromyogram, and body movement electrodes were positioned between 2130 and 2230 and the polyhypnographic recordings started at 2300 . The lights in the laboratory were switched off between 2300 and 0700 . The polyhypnographic recording equipment and the observer were located in a small room adjoining the laboratory, which could be entered without disturbing the subject.

\section{COMBINED OESOPHAGEAL MANOMETRIC AND PH METRIC SYSTEM}

The oesophageal $\mathrm{pH}$ and manometric recording and analysis system ${ }^{46}$ used a measuring assembly consisting of a silicone tube incorporating a combined glass $\mathrm{pH}$ electrode (GK 2801C, Radiometer Copenhagen, Denmark) at the tip and two solid state pressure transducers (MTCP5F; Honeywell), one located at the tip and the other, $10 \mathrm{~cm}$ proximal to the tip. The assembly was connected to a 1 Mbyte solid state data logger (LZ-105mano; Kaufhold Ingenieurbüro, Berlin, Germany) with sampling rates of $0.5 \mathrm{~Hz}$ for $\mathrm{pH}$ data and $5 \mathrm{~Hz}$ for pressure data. After the recording, all data were transferred to the host computer (Atari Mega-ST4; Atari Corporation, Sunnyvale, CA, USA) running under the OS-9 operating system (Microware, DesMoines, IA, USA) for automated analysis ${ }^{4}$ and subsequent back up on longterm storage media.

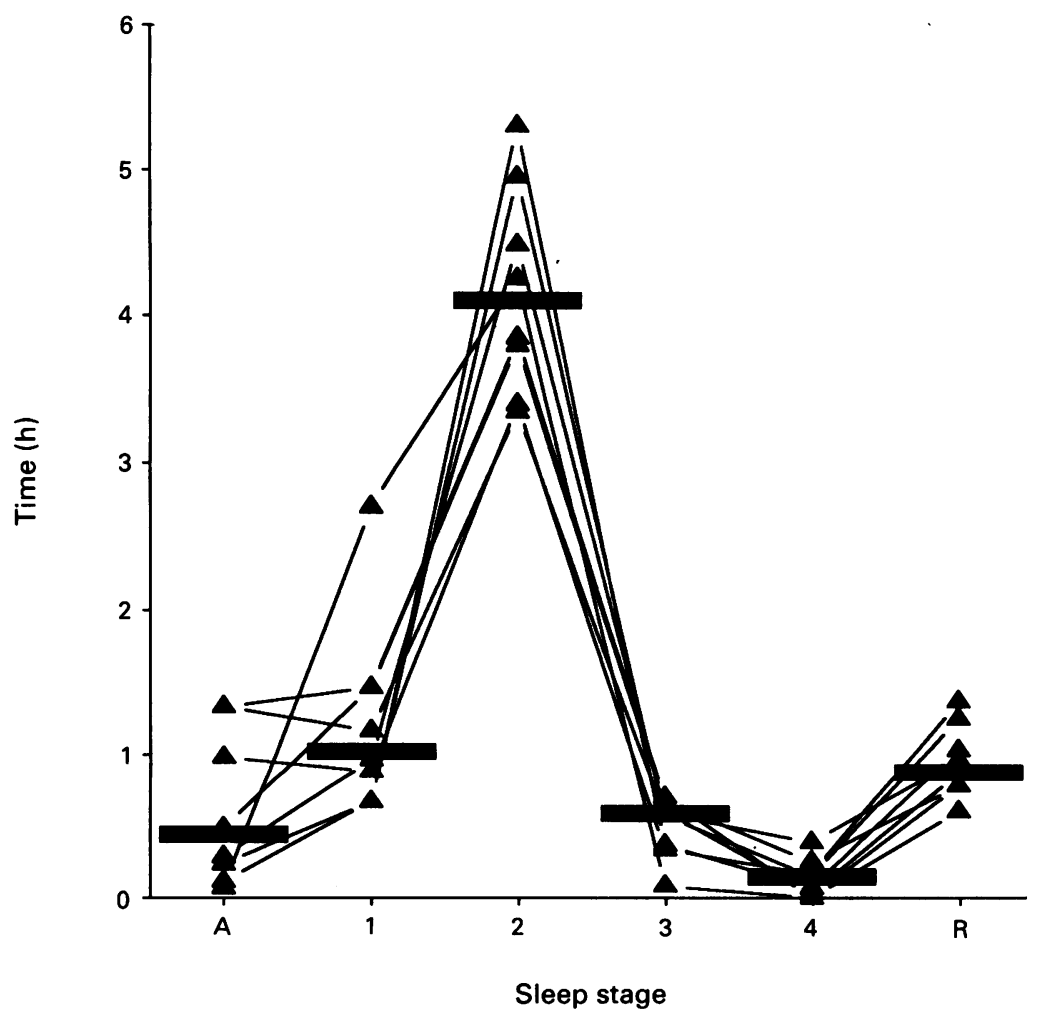

Figure 1: Total time spent by each subject in sleep stages 1 to 4, rapid eye movement sleep $(R)$, and periods of spontaneous arousal $(A)$ from sleep, excluding the waking periods at the start and end of the night. Triangles show individual values for each subject and horizontal bars, the group median values.

\section{ELECTROENCEPHALOGRAPHIC, ELECTRO-} OCULOGRAPHIC, AND ELECTROMYOGRAPHIC RECORDINGS

Continuous all night polyhypnograms were recorded with a Beckman R-411 Dynograph Recorder (Sensormedics, Anaheim, CA, USA), which was calibrated before all recordings. The electroencephalographic recordings were derived from frontal (F7-F8), occipital (O1-O2), and central $(\mathrm{Fz}-\mathrm{Cz}-\mathrm{Pz})$ electrodes according to the 10-20 International System ${ }^{7}$; electrooculographic electrodes were placed $1 \mathrm{~cm}$ lateral to the outer canthi of the eyes (left eye - Al and right eye - A2). Swallow related electromyographic recordings were obtained using two cutaneous electrodes fixed bilaterally in the submental region and body movements were monitored with an acceleration sensor fixed to the left forearm.

\section{DATA ANALYSIS}

The polyhypnograms were synchronised with the oesophageal $\mathrm{pH}$ and manometric recordings to permit combined analysis. The electroencephalographic, electro-oculographic, and body movement recordings were evaluated manually; sleep stages were scored blindly in 30 second epochs. ${ }^{8}$ If body movements were noted in conjunction with the electroencephalographic features of rapid eye movement (REM) sleep, the relevant 30 second epoch was subdivided and only the segment not associated with body movements was registered as a REM phase. The time of onset and duration of the individual sleep stages were noted and used to calculate the overall duration of the respective sleep stages in each subject. Waking periods were subclassified into those that came before the subjects' first period of sleep and 'spontaneous' arousals, which occurred after the subject had first gone to sleep, including the final arousal periods that lasted until the end of the recording at 0700 .

An initial analysis of the oesophageal $\mathrm{pH}$ and manometry recordings determined the total number of gastro-oesophageal reflux episodes (periods of oesophageal $\mathrm{pH}<4$ ) and the total reflux time (percentage of the recording during which $\mathrm{pH}<4)^{910}$ in addition to the number of propagated and non-propagated contractions. ${ }^{4} \mathrm{~A}$ propagated contraction was defined as a contraction detected by the distal pressure transducer no less than one and no more than 10 seconds after its detection by the proximal transducer. All other contractions, whether simultaneous or segmental, were considered to be nonpropagated.

The computerised analysis of the combined electroencephalographic, electro-oculographic, pharyngeal electromyographic, and oesophageal data for the period from 2300 to 0700 allowed the determination of the number, type, and characteristics of all contractions with respect to arousal and the various sleep stages. Oesophageal contractions associated with pharyngeal electromyographic activity were considered to be primary, swallow related contractions; in the absence of electromyographic activity, contractions were considered to be secondary or swallow unrelated. The character- 
Figure 2: Mean time spent hourly by each subject in sleep stages 1 and 2 (crosshatching), sleep stages 3 and 4 (diagonal hatching) and rapid eye movement ( $R E M)$ sleep (solid fill). Subjects were awake for the remainder of the time; subjects were awake for a mean of 13.2 minutes before falling asleep for the first time; thereafter, all periods of wakefulness followed spontaneous arousals.

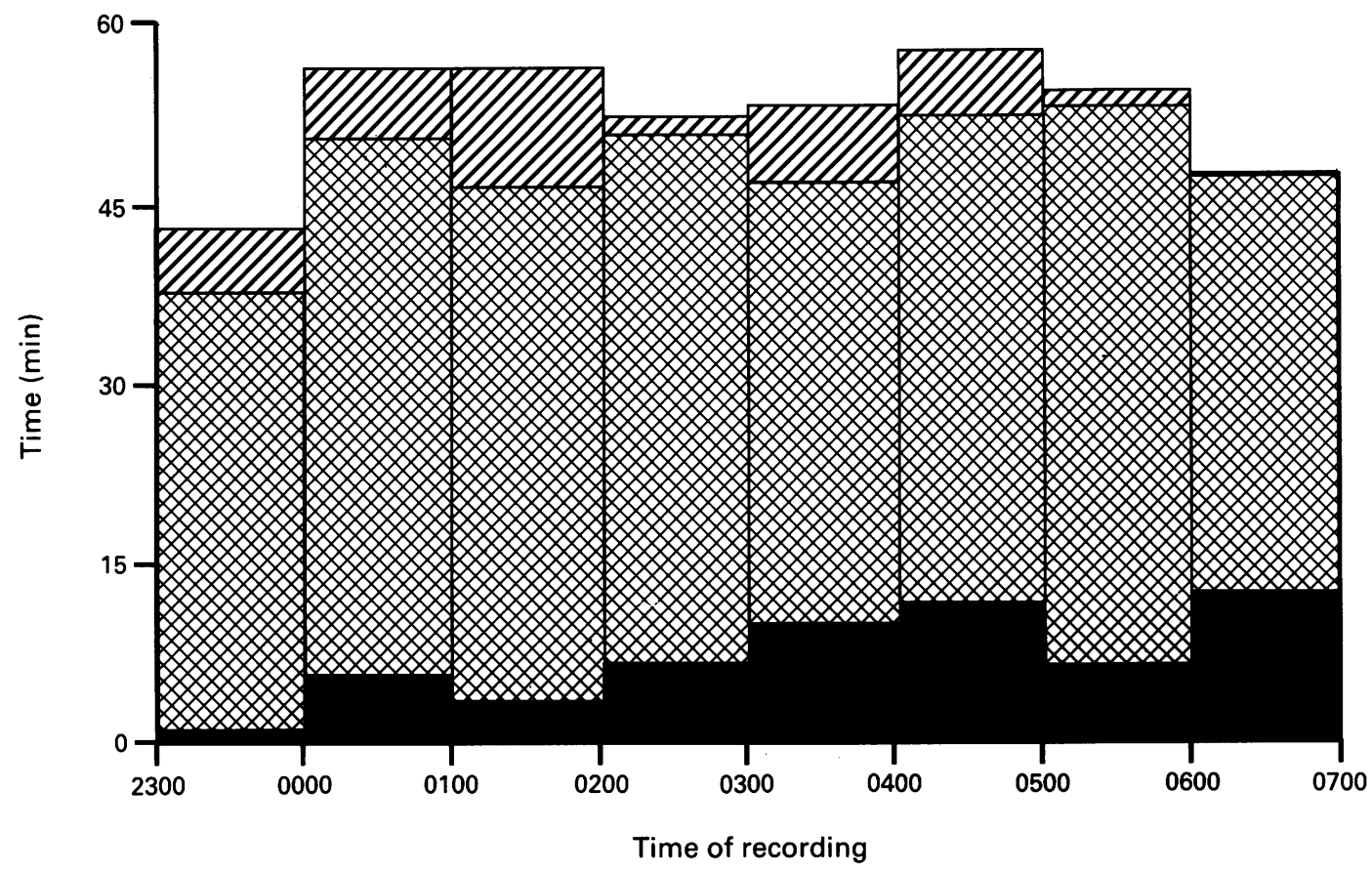

istics of nocturnal contractions were compared with those of daytime contractions during the combined non-meal daytime periods from 2000 to 2200 and 0800 to 1000 . Combined 10 hour printouts (2200 to 0800 ) of the $\mathrm{pH}$ and manometric data, pharyngeal electromyographic events, and the various sleep stages permitted a detailed inspection and analysis of all recordings.

Data are presented as the median and range (minimum to maximum) throughout. Friedman's test, followed by the Wilcoxon-Wilcox location test if appropriate, was used to test the hypotheses that primary contractions were more frequent during the waking state and during stage 1 sleep than during deep (stages 2 to 4 ) and
REM sleep and that secondary contractions were comparably frequent during all sleep stages. An exact version of the Wilcoxon signed rank test ${ }^{11}$ was used in a descriptive analysis to compare contraction characteristics during day and night time periods and also to compare primary and secondary nocturnal propagated contractions with respect to amplitude, duration, area under the curve, and propagation velocity.

\section{Results}

All subjects slept well; stage 2 sleep predominated and spontaneous arousals were short lasting (Fig 1). The mean time taken for the subjects
Figure 3: Representative 10 hour segment of an oesophageal pH and manometric recording from 2200 to 0800 showing the relation between sleep stages and oesophageal activity.

The uppermost panel depicts sleep stages (rapid eye movement sleep represented by solid areas) and the second panel, submentall pharyngeal

electromyographic (EMG) swallowing activity from 2300 to 0700 . In the third and fourth panels, vertical bars show the time of occurrence and amplitude of all contractions $15 \mathrm{~cm}$ and $5 \mathrm{~cm}$, respectively, above the lower oesophageal sphincter (LOS). The fifth panel shows oesophageal $\mathrm{pH}$ as recorded $5 \mathrm{~cm}$ above the LOS.
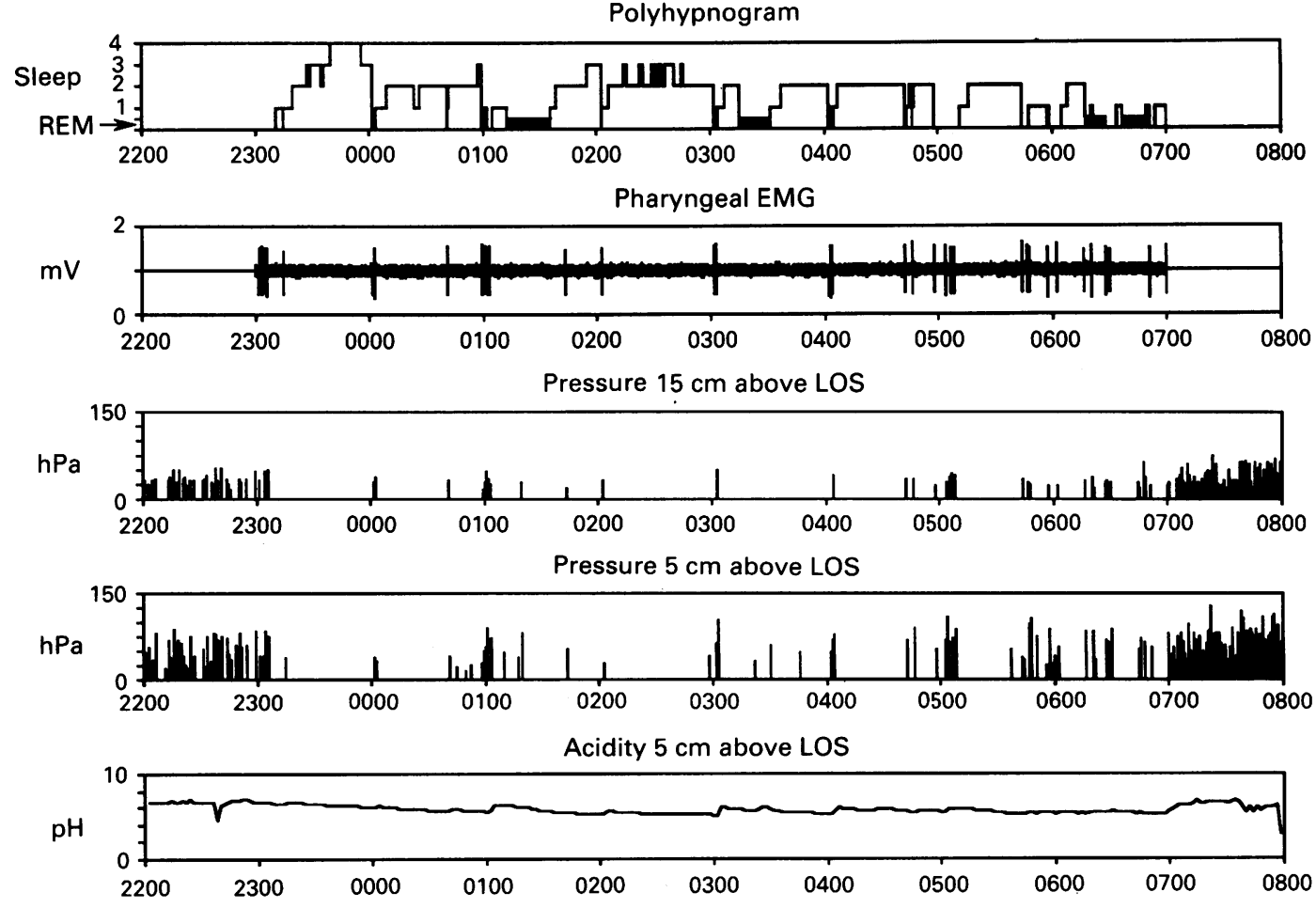


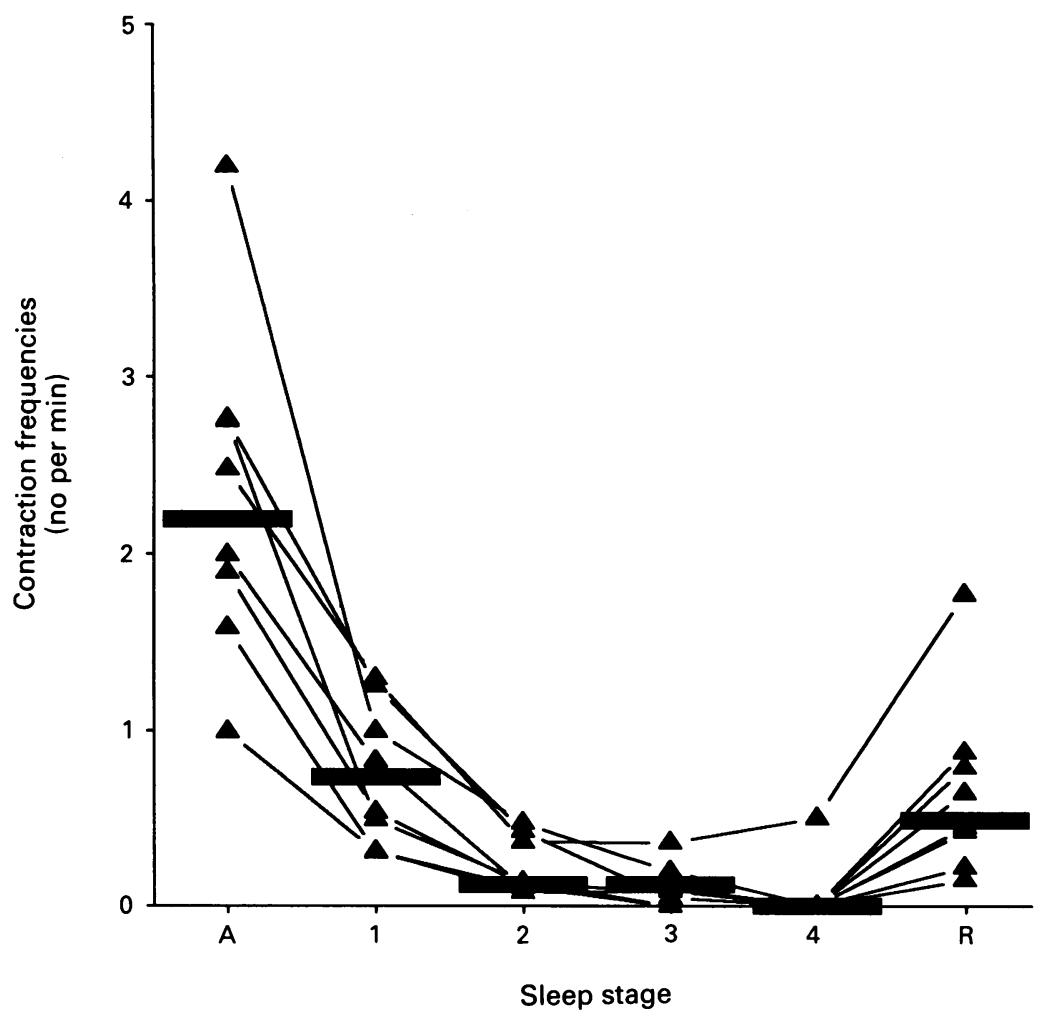

Figure 4: Contraction frequencies per minute for all contractions during periods of spontaneous arousal $(A)$, sleep stages $I$ to 4 and rapid eye movement sleep $(R)$. Triangles show the contraction frequency for each subject and horizontal bars, the group median contraction frequencies.

to fall asleep was $13 \cdot 2 \mathrm{~min}$ but, thereafter, periods of spontaneous arousal lasted a mean of $2 \cdot 3$ to $7 \cdot 1 \mathrm{~min}$ per hour rising to $11 \cdot 3 \mathrm{~min}$ in the final hour from 0600 to 0700 (Fig 2). The proportion of time spent in sleep stages 3 and 4
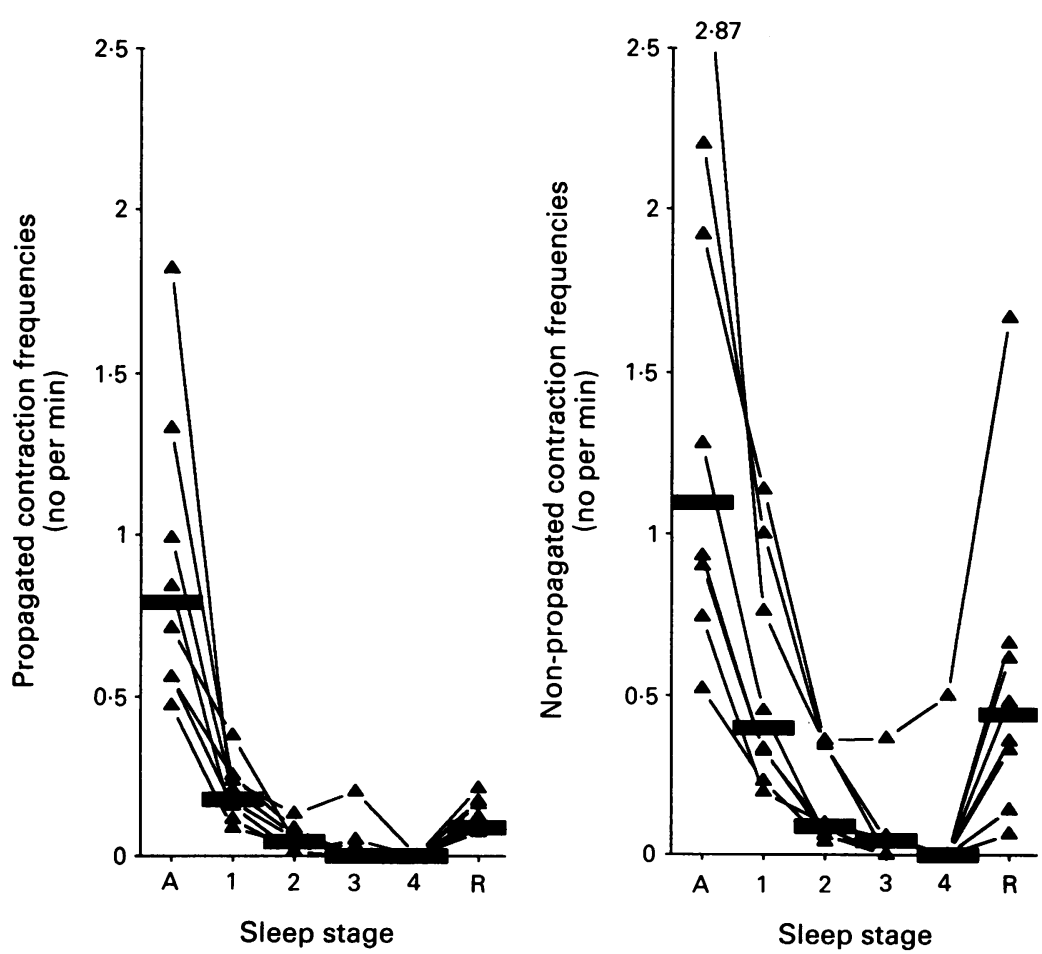

Figure 5: Contraction frequencies per minute for propagated (left panel) and non-propagated contractions (right panel) during periods of spontaneous arousal $(A)$, sleep stages 1 to 4 and rapid eye movement sleep $(R)$. Triangles show the contraction frequency for each subject and horizontal bars, the group median contraction frequencies. declined, while the proportion of each hour spent in REM sleep increased towards morning (Fig 2). The pattern of sleep was similar in all subjects: 45 to $70 \%$ of the night was spent in stage 2, 10 to $15 \%$ in each of the arousal, stage 1 and REM sleep phases, and the remaining 5 to $10 \%$ in stages 3 and 4 combined. The median durations of the separate sleep phases were 2 min (range: 1 to $5.5 \mathrm{~min}$ ) for the arousal periods, $3 \mathrm{~min}(2.5$ to $5 \mathrm{~min})$ for stage $1,12 \mathrm{~min}(7.5$ to $24.5 \mathrm{~min}$ ) for stage $2,3.5 \mathrm{~min}(1.5$ to 12$)$ for stage $3,2 \mathrm{~min}$ ( 0 to $6 \mathrm{~min}$ ) for stage 4 , and $14 \mathrm{~min}$ ( 6 to $25 \mathrm{~min}$ ) for REM sleep.

A representative 10 hour recording (Fig 3) shows a lower contraction frequency during the sleep period ( 2300 to 0700 ) than during the one hour periods of wakefulness before and after sleep; contraction frequency and amplitude were maximal during breakfast (0730 to 0800). Overnight, long periods of oesophageal inactivity were interspersed by bursts of contractions that coincided with arousal, stage 1 and early morning REM sleep; contraction frequencies were low during stage 2 sleep. Pharyngeal electromyographic activity was seen almost exclusively during periods of transient arousal. In the recording shown (Fig 3), as in the other recordings, there were no episodes of gastrooesophageal reflux detected between 2300 and 0700 that might have accounted for the bursts of motor activity.

\section{OESOPHAGEAL pH}

Subjects had a median of 15 (range: 10 to 31) episodes of gastro-oesophageal reflux, almost all of which occurred postprandially. During sleep, two subjects each had one reflux episode: one occurred at the start of the first phase of stage 1 sleep, at 2300, and the second occurred during a transient arousal just before 0600 . In each case, contraction frequencies were higher during the four minute period extending from two minutes before to two minutes after the onset of reflux than they were during the remainder of the night: $2 \cdot 7 v 0.32$ contractions $/ \mathrm{min}$ in one subject and $3.5 v 0.58$ contractions $/ \mathrm{min}$ in the other subject. Primary contractions constituted $67 \%$ of all reflux related contractions; this was comparable with the percentage of contractions that were swallow related during periods of wakefulness (see below).

\section{OESOPHAGEAL MOTILITY}

The overall contraction frequency during nonmeal daytime periods (median: $1.63 / \mathrm{min}$; range: 1.01 to 1.98 ) was lower than during transient nocturnal arousal periods (Fig 4) although the frequency of daytime propagated contractions (median: $0.65 / \mathrm{min}$; range: 0.37 to 0.86 ) was similar to that of nocturnal propagated contractions (Fig 5). The frequency of all contractions (Fig 4), both propagated and non-propagated, decreased considerably in stage 1 sleep and continued to decrease in stages 2 to 4 (Fig 5). Stages 3 and 4 constituted only a small part of the recording and, as they were associated with very low contraction frequencies, they were combined with stage 2 for all subsequent analyses. 

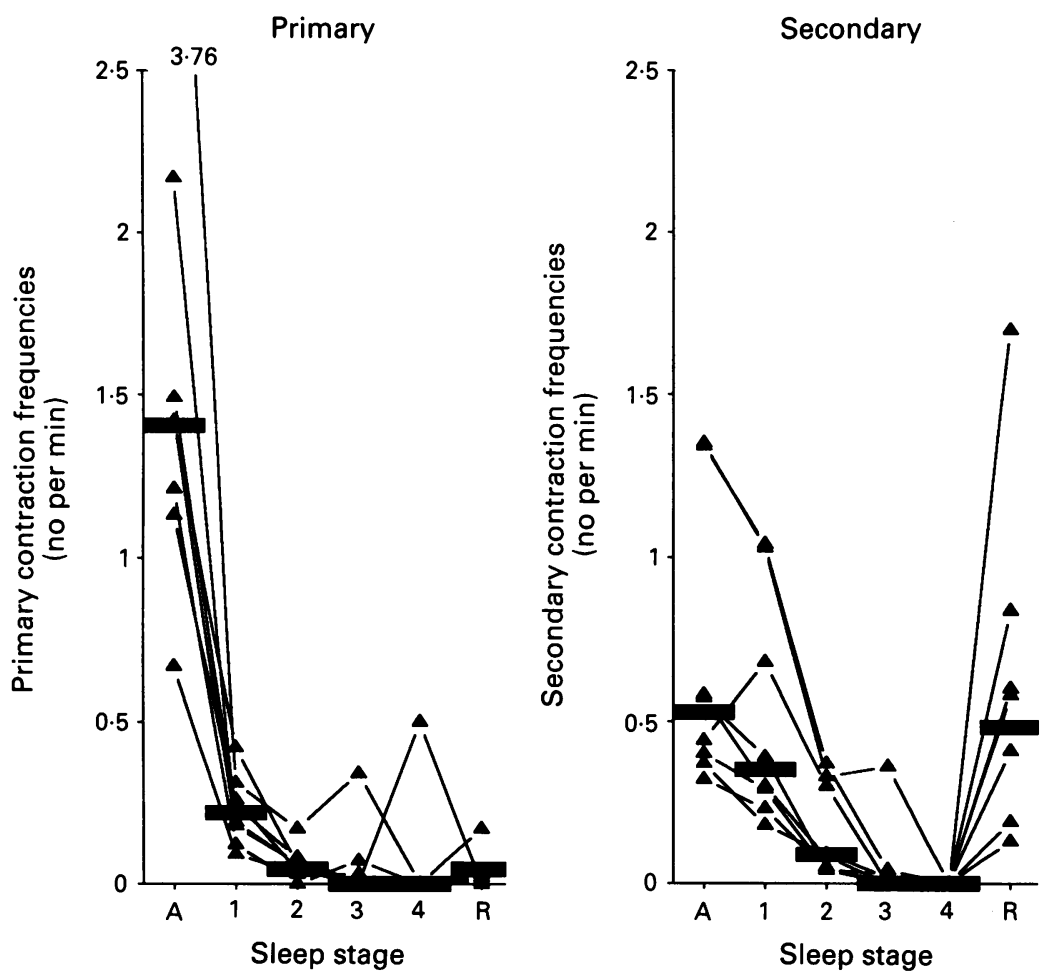

Figure 6: Contraction frequencies per minute for primary (left panel) and secondary contractions (right panel) during periods of spontaneous arousal $(A)$, sleep stages 1 to 4 and rapid eye movement sleep $(R)$. Triangles show the contraction frequency for each subject and horizontal bars, the group median contraction frequencies.

During REM sleep, the frequencies of both propagated and non-propagated contractions increased to those seen during stage 1 . During the day, $38 \%$ (range: 34 to $49 \%$ ) of all con-

TABLE I Median number (range) of primary and secondary contractions per minute during different sleep stages

\begin{tabular}{|c|c|c|c|c|}
\hline \multirow{2}{*}{$\begin{array}{l}\text { Contraction } \\
\text { (no/min) }\end{array}$} & \multicolumn{4}{|l|}{ Sleep stages } \\
\hline & Awake & 1 & $2,3,4$ & $R E M$ \\
\hline Primary & $1 \cdot 42$ & $\begin{array}{l}0.22 \\
\quad(0.09-0.42)\end{array}$ & 0.05 & $\begin{array}{l}0.03 \\
\quad(0.00-0.17)\end{array}$ \\
\hline Secondary & 0.51 & $\begin{array}{l}0.35 \\
(0 \cdot 18-1.04)\end{array}$ & 0.08 & $\begin{array}{l}0.50 \\
\quad(0 \cdot 13-1 \cdot 70)\end{array}$ \\
\hline
\end{tabular}

Statistically significant differences between contraction frequencies in the various sleep stages are shown by ${ }^{\star}(\mathrm{p}<0 \cdot 05)$ and $\star \star$
movement.

TABLE II Propagated contraction characteristics during day and night time periods

\begin{tabular}{|c|c|c|c|c|}
\hline \multirow[b]{3}{*}{ Contraction variable } & \multicolumn{4}{|l|}{ Time period } \\
\hline & \multirow[b]{2}{*}{ Daytime total } & \multirow[b]{2}{*}{ Night time total } & \multicolumn{2}{|l|}{ Night time } \\
\hline & & & Primary & Secondary \\
\hline Amplitude (hPa) & $\begin{array}{l}54 \cdot 3 \\
(36 \cdot 2-79 \cdot 4)\end{array}$ & $\begin{array}{c}68 \cdot 0 \\
(41 \cdot 0-93.9) \\
-p=0 \cdot 109-\mid\end{array}$ & $\begin{array}{l}76 \cdot 0 \\
(46 \cdot 5-108)\end{array}$ & $\begin{array}{c}51.9 \\
(37 \cdot 7-67 \cdot 2) \\
-p=0.00781-\end{array}$ \\
\hline Duration (s) & $\begin{array}{l}3 \cdot 16 \\
(2 \cdot 53-3 \cdot 59)\end{array}$ & $\begin{array}{l}3.65 \\
(2 \cdot 95-4 \cdot 10) \\
n=0.0234--\end{array}$ & $\begin{array}{l}4 \cdot 06 \\
(3 \cdot 26-5 \cdot 28)\end{array}$ & $\begin{array}{c}3.08 \\
(2.88-3.62) \\
-n=0.00781-1\end{array}$ \\
\hline $\begin{array}{l}\text { Area under curve } \\
(\mathrm{hPa} / \mathrm{s})\end{array}$ & 100 & $\begin{array}{c}146 \\
(74 \cdot 8-236) \\
p=0 \cdot 0156-1\end{array}$ & $185(105-304)$ & $\begin{array}{c}101 \\
(65 \cdot 7-144)\end{array}$ \\
\hline $\begin{array}{l}\text { Propagation velocity } \\
(\mathrm{cm} / \mathrm{s})\end{array}$ & $(2 \cdot 72-3 \cdot 24)$ & $\begin{array}{c}2.61 \\
(2 \cdot 44-3.68) \\
-p=0.601-1\end{array}$ & $\begin{array}{l}2 \cdot 66 \\
(2 \cdot 27-2 \cdot 98)\end{array}$ & $\begin{array}{c}2.75 \\
(1.91-3.95) \\
\mathrm{p}=0.250-1\end{array}$ \\
\hline
\end{tabular}

Median values (ranges) are presented for each time period. Comparisons were made between day and night time periods and between primary and secondary contractions during night time: two-sided $p$ values, calculated using an exact version of the Wilcoxon matched pairs signed rank test, ${ }^{11}$ are shown. values, calculated using an exact version of the Wilcoxon matched pairs signed
p Values smaller than 0.05 were considered to indicate a significant difference. tractions were propagated; this proportion remained comparatively constant during transient arousals (median: 42\%; range: 20 to $66 \%$ ), stage 1 (median: $31 \%$; range: 12 to $46 \%$ ), stages 2 to 4 (median: $33 \%$; range: 3 to $52 \%$ ), and REM sleep (median: $25 \%$; range: 5 to $57 \%$ ); nonpropagated contractions were predominantly distal segmental events detected $5 \mathrm{~cm}$ above the lower oesophageal sphincter.

Primary and secondary contractions could be differentiated only during the nocturnal period when swallow related electromyographic data were available. Primary contractions decreased considerably in frequency once the subjects entered stage 1 sleep; further decreases were seen in stages 2, 3 and 4 combined, and in REM sleep (Fig 6). Nocturnal arousals, stage 1 sleep, and REM sleep were associated with comparable frequencies of secondary contractions that were, for all three periods, higher than the frequency of secondary contractions during stages 2,3 and 4 combined (Table I). During arousal, 74\% (range: 50 to $90 \%$ ) of all contractions were primary; their frequency decreased to $35 \%$ (range: 15 to $52 \%$ ) in stage $1,40 \%$ (range: 0 to $67 \%$ ) in stages 2 to 4 , and $6 \%$ (range: 0 to $23 \%$ ) during REM sleep. Of the secondary, swallow unrelated contractions, a median of $12 \%$ (range: 3.3 to $17 \cdot 4 \%$ ) were propagated whereas $66 \%$ (range: 39 to $85 \%$ ) of swallow related contractions were propagated.

The duration and the area under the curve of nocturnal propagated contractions were generally greater than those of non-meal daytime contractions (Table II) but neither the nocturnal amplitude nor the nocturnal propagation velocity differed significantly from their respective daytime values. During the night, primary contractions had a greater amplitude, duration, and area under the curve than secondary contractions; the contraction types did not differ with respect to propagation velocity (Table II). Overall, the nocturnal period contained higher proportions of high amplitude $(>200 \mathrm{hPa})$ and prolonged ( $>10 \mathrm{~s}$ ) contractions, typified by Figure 7, than did the non-meal, daytime periods (Table III).

\section{Discussion}

The results of this study show clearly that nocturnal oesophageal motor activity is sleep stage dependent. The first hypothesis - that the frequency of primary contractions would diminish progressively in the deeper sleep stages - is supported by these findings. In contrast, the second hypothesis may be rejected: secondary contractions also decreased slightly during light sleep, with a considerable decrease during stages 2,3 and 4 but, unlike primary contractions, they were as frequent during REM sleep as during periods of arousal.

The durations of the different sleep stages were similar in all volunteers although the total duration of REM sleep was shorter than in previous reports, ${ }^{12}$ probably because it was decided that body movements should preclude the classification of an epoch as REM sleep. This would also explain the low incidence of REM related primary contractions as REM related 
Figure 7: A two minute segment of a nocturnal oesophageal $\mathrm{pH}$ and manometric recording illustrating an abnormally prolonged, high amplitude, multipeaked contraction (circled) unrelated to an episode of gastro-

oesophageal reflux, which was recorded $5 \mathrm{~cm}$ above the lower oesophageal sphincter (LOS) during rapid eye movement sleep.
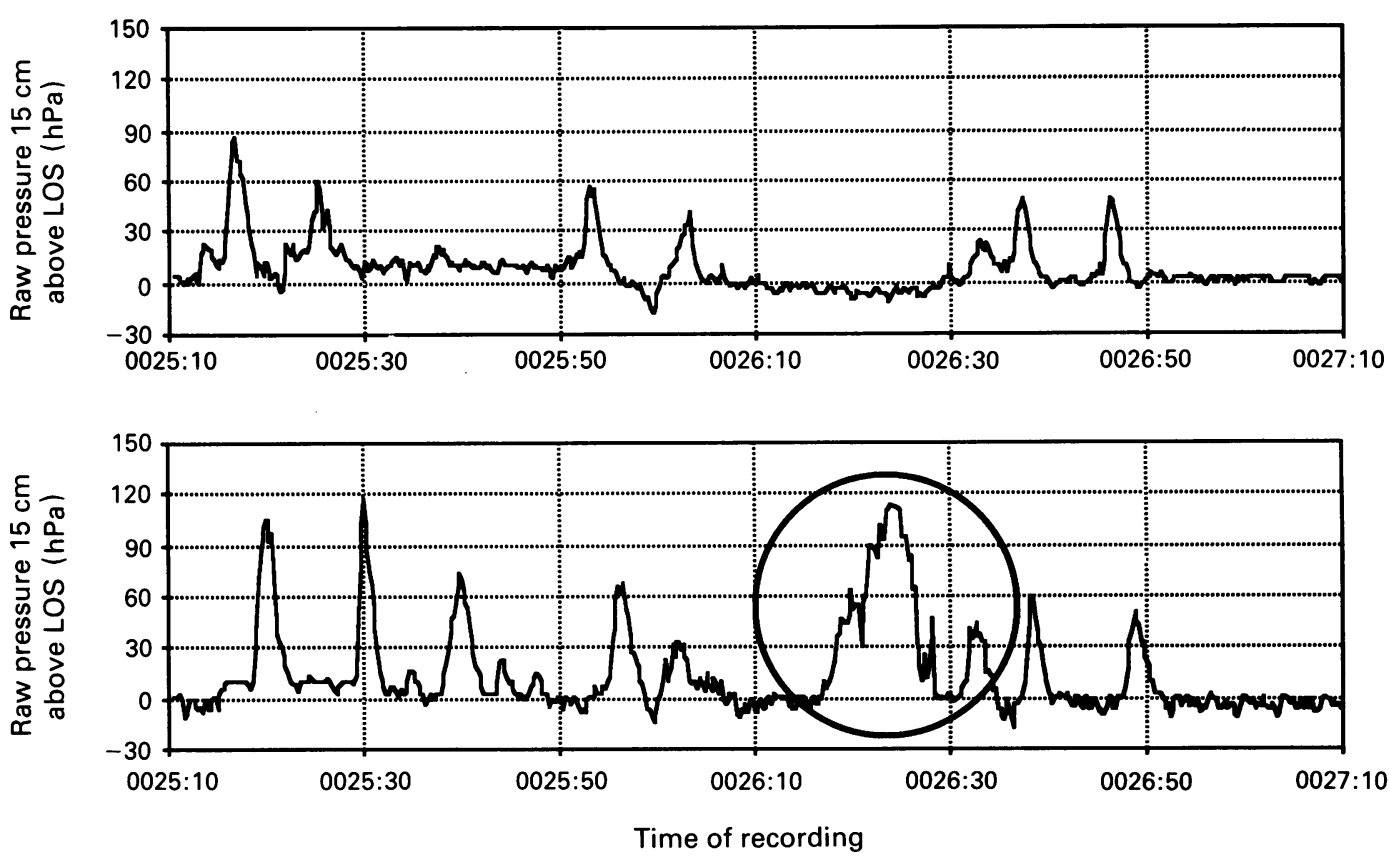

swallowing activity has been reported to be associated strongly with movement arousals. ${ }^{13}$ This study examined the incidence of swallowing activity only in so far as it related to oesophageal contractions; thus, it is possible that REM sleep is associated, none the less, with an increased incidence of abortive swallows.

As in previous studies, ${ }^{14}$ 15 there were long periods of nocturnal oesophageal motor quiescence interspersed by bursts of contractions. It is possible that these contraction bursts were caused by episodes of gastro-oesophageal reflux or that they were related to the onset of migration motor complexes in the upper gastrointestinal tract. Contraction bursts, however, were seen in all subjects despite the fact that only two gastrooesophageal reflux episodes were seen between 2300 and 1700 in the eight recordings. It, therefore, seems unlikely that gastro-oesophageal reflux was an important stimulant of oesophageal motility in these healthy subjects, although it is conceivable that the contraction bursts were provoked by episodes of gastrooesophageal reflux that were not detected by the $\mathrm{pH}$ electrode $5 \mathrm{~cm}$ above the lower oesophageal sphincter. The lower oesophageal sphincter, and possibly the distal oesophageal body, may participate in up to $50 \%$ of migrating motor complexes. This study did not examine migrating motor complex activity directly but the results do suggest that oesophageal activity is

TABLE III Cumulative numbers of 'atypical' (amplitude $>200 \mathrm{hPa}$ or duration $>10 \mathrm{~s}$ ) and normal (amplitude $<200$ hPa or duration $<10 \mathrm{~s}$ ) contractions recorded during daytime and night time periods in all subjects

\begin{tabular}{lccc}
\hline \multirow{4}{*}{$\begin{array}{l}\text { Contraction } \\
\text { characteristics }\end{array}$} & No of contractions & & \\
\cline { 2 - 4 } & Daytime & Night time & $p^{\star}$ \\
\hline Amplitude: & $20(0 \cdot 43 \%)$ & $23(0 \cdot 87 \%)$ & 0.0244 \\
$>200 \mathrm{hPa}$ & 4677 & 2619 & \\
$<200 \mathrm{hPa}$ & $31(0.66 \%)$ & $31(1 \cdot 2 \%)$ & 0.0239 \\
$\begin{array}{c}\text { Duration: } \\
>10 \mathrm{~s}\end{array}$ & 4666 & 2611 & \\
$<10 \mathrm{~s}$ & & & \\
\hline
\end{tabular}

^Fisher's exact test ( 2 sided): night time $v$ daytime. related to sleep stages. Despite evidence to suggest a relation between REM sleep and the onset of duodenal migrating motor complexes, ${ }^{17}$ no clear correlation has been established between sleep and the occurrence of migrating motor complexes $^{18}$ and as, furthermore, oesophageal activity seen in this study did not show the 60 to 90 minute periodicity characteristic of migrating motor complexes, it seems unlikely that most oesophageal contraction clusters are migrating motor complex related.

The frequency of oesophageal contractions differs considerably between sleep stages with an appreciable decrease from stage 1 to stage 2 sleep. The nocturnal motility pattern - long periods of oesophageal motor inactivity interspersed by bursts of contractions - seen in previous manometric studies ${ }^{615}$ is probably attributable, in part, to prolonged periods of stage 2 sleep, associated with stages 3 and 4 , during which centrally originating stimulation of oesophageal contractions is virtually absent. Contraction bursts seem to depend on two different mechanisms: swallowing, shown by pharyngeal electromyographic activity, leading to primary, propagated contractions is responsible for motor activity during periods of short term arousal. On the other hand, secondary contractions and non-propagated contractions, unrelated to swallowing, are generally of lower amplitude and shorter duration than primary contractions and are more typical of the somewhat longer REM sleep phases despite the occurrence of occasional prolonged, high amplitude contractions during REM sleep (Table III, Fig 7).

The high rate of secondary contractions during REM sleep raises two possibilities with regard to the control of oesophageal motor activity. On the one hand, it is possible that the oesophagus is intrinsically active and that it is usually subject to a tonic inhibitory input; abolition of this inhibitory input during REM sleep would then allow the oesophagus to contract in a disorganised, non-propagative manner. 
Secondary contractions are virtually absent, however, during sleep stages 2,3 and 4 suggesting that the oesophagus is not, like more distal parts of the gastrointestinal tract, intrinsically active and, thus, potentially autonomous. Alternatively, secondary contractions seen during REM sleep may be related directly to REM associated changes in sympathetic and parasympathetic nervous activity, ${ }^{19}$ which have been implicated in the large variations in heart rate and other cardiorespiratory functions seen during REM sleep. ${ }^{1920}$ The lower amplitude, smaller area under the curve, and shorter duration of secondary contractions compared with primary contractions implies that the two contraction types may be controlled differently as well as being initiated differently. Because secondary contractions are not initiated voluntarily or in response to pharyngeal stimuli, they may be less likely to respond to a luminal bolus by increasing contraction amplitude or duration.

The findings that prolonged or high amplitude contractions are more likely at night than during the non-meal daytime and that primary nocturnal contractions have a higher amplitude and a greater area under the curve than daytime contractions should be borne in mind when interpreting longterm manometric recordings, particularly if the person is being investigated for non-cardiac chest pain. Because, in this study, several healthy volunteers developed both prolonged and high amplitude contractions, predominantly during REM sleep, the occurrence of apparently abnormal or pathological contraction types (Table III, Fig 7) at night should not be considered sufficient to make a diagnosis of oesophageal dysmotility, particularly if the subject has no symptoms related temporally to the contractions. These apparently abnormal contractions may rather be a manifestation of heightened arousal and increased autonomic nervous system activity mentioned previously. ${ }^{1} 1920$

The considerable sleep related variations in oesophageal motility recorded in this study provide a basis for studying the roles of central and peripheral control mechanisms in oesophageal motility disorders, including gastro-oesophageal reflux disease. On line analysis of sleep electroencephalographic recordings ${ }^{21}$ is now available and, in conjunction with oesophageal $\mathrm{pH}$ and manometry recordings, it would allow local stimulation of the oesophagus by balloon distension, ${ }^{22}$ acid perfusion ${ }^{23}$ or electrical stimulation ${ }^{24}$ during specific sleep stages. Study of the oesophageal motor response and also, possibly, the cortical evoked potentials would help to determine the roles of local stimuli, a subject's responsiveness to such stimuli and other central nervous system factors in initiating and modifying oesophageal motility. Similarly, an understanding of the cause of 'atypical', prolonged, high amplitude oesophageal contractions could lay the foundation for a better understanding of the pathogenesis of conditions such as diffuse oesophageal spasm.

The study was supported by grants from the Swiss National Foundation (SNF 32-26369.89) and the Deutsche Forschungsgemeinschaft (DFG Em 36/2-1)

These data were presented, in part, at the 6th European Symposium on Gastrointestinal Motility in Augsburg, June 1990 and published in abstract form in the fournal of Gastrointestinal Motility 1990; 2: 131 and 2: 136.

1 Anderson KO, Dalton CB, Bradley LA, Richter JE. Stress induces alteration of esophageal pressures in healthy volunteers and non-cardiac chest pain patients. Dig Dis Sci 1989; 34: 83-91.

2 Diamant NE. Physiology of esophageal motor function. Gastroenterol Clin North Am 1989; 18: 179-94.

3 Arndorfer RC, Stef JJ, Dodds WJ, Linehan J, Hogan WJ. Improved infusion system for intraluminal esophageal manometry. Gastroenterology 1977; 73: 23-7.

4 Emde C, Armstrong D, Bumm R, Kaufhold H-J, Riecken EO, Blum AL. Twenty-four hour continuous ambulatory measurement of oesophageal $\mathrm{pH}$ and pressure: a digital recording system and computer aided manometry analysis. fournal of Ambulatory Monitoring 1990; 3: 47-62.

5 Bumm R, Emde C, Armstrong D, Bauerfeind P, Blum AL. Ambulatory oesophageal manometry: comparison of expert and computer-aided analyses. Fournal of Gastrointestinal Motility 1990; 2: 216-23.

6 Emde C, Armstrong D, Castiglione F, Cilluffo T, Riecken EO, Blum AL. Reproducibility of long-term ambulatory esophageal combined $\mathrm{pH} /$ manometry. Gastroenterology 1991; 100: 1630-7.

7 Jasper $\mathrm{HH}$. The 10-20 electrode system of the International Federation. Electroencephalogr Clin Neurophysiol 1958; 10: 371-5.

8 Rechtschaffen A, Kales A, eds. Berger RJ, Dement WC, Jacobson A, Johnson LC, Jouvet M, Monroe JL, et al. A manual of standardized terminology, techniques and scoring
system for sleep stages of human subjects (Publication No 204). Washington, DC: Public Health Service, US Government Printing Office, 1968.

9 Emde C, Garner A, Blum AL. Technical aspects of intraluminal pH-metry in man: current status and recommendaluminal pH-metry in man: cur
tions. Gut 1987; 28: 1177-88.

10 Emde C, Cilluffo T, Bauerfeind P, Blum AL. Combined esophageal and gastric pH-metry in healthy volunteers. Influence of cable through LES and effect of misoprostol Dig Dis Sci 1989; 34: 79-82.

11 Streitberg B, Röhmel J. Exakte Verteilungen für Rang- und Randomisierungstests im allgemeinen c-Stichprobenproblem. EDV in Medizin und Biologie 1987; 18: $12-9$.

12 West $P$, Kryger $M H$. Sleep and respiration: terminology and methodology. Clin Chest Med 1985; 6: 691-712.

13 Lichter I, Muir RC. The pattern of swallowing during sleep. Electroencephalogr Clin Neurophysiol 1975; 38: 427-32.

14 Armstrong D, Emde C, Bumm R, Castiglione F, Cilluffo T, Blum AL. Twenty-four-hour pattern of esophageal motility Blum AL. Twenty-four-hour pattern of esophageal motility
in asymptomatic volunteers. Dig Dis Sci 1990; 35: 1190-7.

15 Smout AJPM, Breedijk M, van der Zouw C, Akkermans LMA. Physiological gastroesophageal reflux and esophageal motor activity studied with a new system for 24-hour recording and automated analysis. Dig Dis Sci 1989; 34: 372-8

16 Kellow JE, Borody TJ, Phillips SF, Tucker RL, Haddad AC. Human interdigestive motility: variations in patterns from oesophagus to colon. Gastroenterology 1986; 91: 386-95.

17 Finch $P$, Ingram $D$, Henstridge J, Catchpole $B$. The relationship of sleep stage to the migrating gastrointestinal complex of man. In: Christensen J, ed. Gastrointestinal Motility. New York: Raven Press, 1980: 261-5.

18 Kumar D, Idzikowski C, Wingate DL, Soffer EE, Thompson P, Siderfin C. Relationship between enteric migrating motor P, Siderfin C. Relationship between enteric migrating motor complex

19 Zemaityte D, Varoneckas G, Sokolov E. Heart rhythm control during sleep. Psychophysiology 1984; 21: 279-89.

20 Snyder F, Hobson JA, Morrison DF, Goldfrank F. Changes in respiration, heart rate and systolic blood pressure in human sleep. F Appl Physiol 1964; 19: 417-22.

21 Samson-Dollphus D, Delapierre G, Nogues B, Bertoldi I Sleep organisation in children at risk for sudden infant death syndrome. Sleep 1988; 11: 277-85.

22 Castell DO, Wood JD, Frieling T, Wright FS, Vieth RF Cerebral electrical potentials evoked by balloon distension of the human esophagus. Gastroenterology 1990; 98: 662-6.

the human esophagus. Gastroenterology 1990; 98: 662-6.
23 Orr WC, Johnson LF, Robinson MG. Effect of sleep on swallowing, esophageal peristalsis and acid clearance. Gastroenterology 1984; 86: 814-9.

24 Frieling T, Enck P, Wienbeck M. Cerebral responses evoked by electrical stimulation of the esophagus in normal subjects. Gastroenterology 1989; 97: 475-8. 